\title{
Bipolar polaron pair recombination in polymer/fullerene solar cells
}

Kupijai, Alexander J.; Behringer, Konstantin M.; Schaeble, Florian G.; Galfe, Natalie E.; Corazza, Michael; Gevorgyan, Suren A.; Krebs, Frederik C; Stutzmann, Martin; Brandt, Martin S.

Published in:

Physical Review B

Link to article, DOI:

10.1103/physrevb.92.245203

Publication date:

2015

Document Version

Publisher's PDF, also known as Version of record

Link back to DTU Orbit

Citation (APA):

Kupijai, A. J., Behringer, K. M., Schaeble, F. G., Galfe, N. E., Corazza, M., Gevorgyan, S. A., Krebs, F. C., Stutzmann, M., \& Brandt, M. S. (2015). Bipolar polaron pair recombination in polymer/fullerene solar cells. Physical Review B, 92(24), [245203]. https://doi.org/10.1103/physrevb.92.245203

\section{General rights}

Copyright and moral rights for the publications made accessible in the public portal are retained by the authors and/or other copyright owners and it is a condition of accessing publications that users recognise and abide by the legal requirements associated with these rights.

- Users may download and print one copy of any publication from the public portal for the purpose of private study or research.

- You may not further distribute the material or use it for any profit-making activity or commercial gain

- You may freely distribute the URL identifying the publication in the public portal 


\title{
Bipolar polaron pair recombination in polymer/fullerene solar cells
}

\author{
Alexander J. Kupijai, ${ }^{1,}{ }^{*}$ Konstantin M. Behringer, ${ }^{1}$ Florian G. Schaeble, ${ }^{1}$ Natalie E. Galfe, ${ }^{1}$ Michael Corazza, ${ }^{2}$ \\ Suren A. Gevorgyan, ${ }^{2}$ Frederik C. Krebs, ${ }^{2}$ Martin Stutzmann, ${ }^{1}$ and Martin S. Brandt ${ }^{1}$ \\ ${ }^{1}$ Walter Schottky Institut and Physik-Department, Technische Universität München, Am Coulombwall 4, 85748 Garching, Germany \\ ${ }^{2}$ Department of Energy Conversion and Storage, Technical University of Denmark, Frederiksborgvej 399, 4000 Roskilde, Denmark
}

(Received 7 October 2015; revised manuscript received 26 November 2015; published 17 December 2015)

\begin{abstract}
We present a study of the rate-limiting spin-dependent charge-transfer processes in different polymer/fullerene bulk-heterojunction solar cells at $10 \mathrm{~K}$. Observing central spin-locking signals in pulsed electrically detected magnetic resonance and an inversion of Rabi oscillations in multifrequency electron-double-resonance spectroscopy, we find that the spin response of both spin-coated and printed P3HT/PCBM and spin-coated PCDTBT/PCBM solar cells at low temperatures is governed by bipolar polaron pair recombination and quantitatively determine the polaron-polaron coupling strength with double electron-electron resonance experiments. Furthermore spin Hahn echo decay and inversion recovery measurements are performed to measure spin coherence and recombination times of the polaron pairs, respectively.
\end{abstract}

DOI: 10.1103/PhysRevB.92.245203

PACS number(s): 33.40.+f, 72.20.Jv, 88.40.jr

\section{INTRODUCTION}

The low spin-orbit coupling of organic semiconductors and the resulting long spin lifetimes lead to a pronounced spin dependence of charge-transport processes, providing an opportunity for further improvements of the efficiency of light-emitting diodes (LEDs) [1] and solar cells [2,3] as well as for the development of spintronic devices [4,5]. Magnetic resonance methods are uniquely suited to study the influence of spins on electronic transport on time scales as fast as $100 \mathrm{~ns}$, often allowing to microscopically identify relevant or rate-limiting processes. The electrical detection of magnetic resonance in polymers and fullerenes goes back to at least 1978 and 1993, respectively [6-11].

In organic semiconductors, these methods should therefore be particularly helpful to understand the physics of polarons. Polarons are the elementary charged excitations in both small organic molecules and polymers, where two different fundamental spin-dependent processes involving polarons can occur, as sketched in Fig. 1: bipolar recombination of a polaron pair $[12,13]$ and unipolar hopping, where a doubly charged bipolaron is created from two like-charged polarons [14-16]. While the former process gives rise to spin-dependent changes in the charge or, rather, the polaron carrier concentration, the latter results in a spin-dependent change of polaron mobility. Which of the two polaronic processes is observed in organic diodes such as LEDs and solar cells, where two different polarons are present, is still discussed intensively $[5,17,18]$. The distinction is particularly difficult in the case where the spectroscopic signatures of positive and negative polarons are very similar, as in the case of poly( $p$-phenylene-vinylene) (PPV) [15,19-21]. A more direct assignment should be possible in organic devices where the two polarons can be distinguished spectroscopically. This would also allow the direct transfer of pulse sequences, developed for the study of charge transport and recombination in inorganic semiconductors such as amorphous and crystalline Si [22-25], to the investigation of organic semiconductors. Here we study polaron transport

\footnotetext{
*alexander.kupijai@wsi.tum.de
}

and kinetics in two topical organic bulk heterostructures $[26,27]$, fabricated by both spin coating and printing, with the help of light-induced electron paramagnetic resonance (LEPR) and continuous-wave and, in particular, pulsed electrically detected resonance (cwEDMR and pEDMR, respectively) $[23,28]$. Using spin locking and multifrequency magnetic resonance spectroscopy, we identify bipolar recombination of polaron pairs as the dominant spin-dependent process in bulk heterojunctions at low temperature and under illumination and determine polaron life and coherence times.

\section{EXPERIMENTAL DETAILS}

\section{A. Samples}

The study presented in this work was performed on bulk heterojunction organic solar cells consisting of poly(3-hexylthiophene-2,5-diyl) (P3HT) or poly[ $N-9^{\prime}$ heptadecanyl-2,7-carbazole-alt-5, 5-(4', 7'-di-2-thienyl-2' $1^{\prime}$, $3^{\prime}$-benzothiadiazole)] (PCDTBT) polymers as donor material and phenyl- $\mathrm{C}_{61}$-butyric acid methyl ester (PCBM) fullerenes as the acceptor.

The spin-coated solar cells were produced on lithographically structured indium tin oxide (ITO)-on-glass substrates with a dimension of approximately $4 \times 40 \mathrm{~mm}^{2}$ in order to fulfill geometric requirements of the spectrometer. A hole transport layer of poly(3,4-ethylenedioxythiophene) : polystyrene sulfonate (PEDOT: PSS) was spin coated on the substrate first. The spin coating of the active blend (P3HT: $\mathrm{PCBM}=1: 1$, PCDTBT: PCBM $=1: 2)$ at $720 \mathrm{rpm}$, which results in a thickness of around $100 \mathrm{~nm}$, was performed under inert gas conditions in an argon glove box. Finally, a top electrode [approximately $120 \mathrm{~nm}$ aluminum (P3HT/PCBM cells), $1 \mathrm{~nm}$ calcium and $120 \mathrm{~nm}$ aluminum (PCDTBT/PCBM cells)] was evaporated inside the glove box. The active area of the actual cell is about $4 \mathrm{~mm}^{2}$; the rest of the substrate is needed to electrically contact the cell without disturbing the microwave resonator. Without further encapsulation the samples were bonded onto a sample holder and transferred into the spectrometer, exposing the samples to air in darkness 


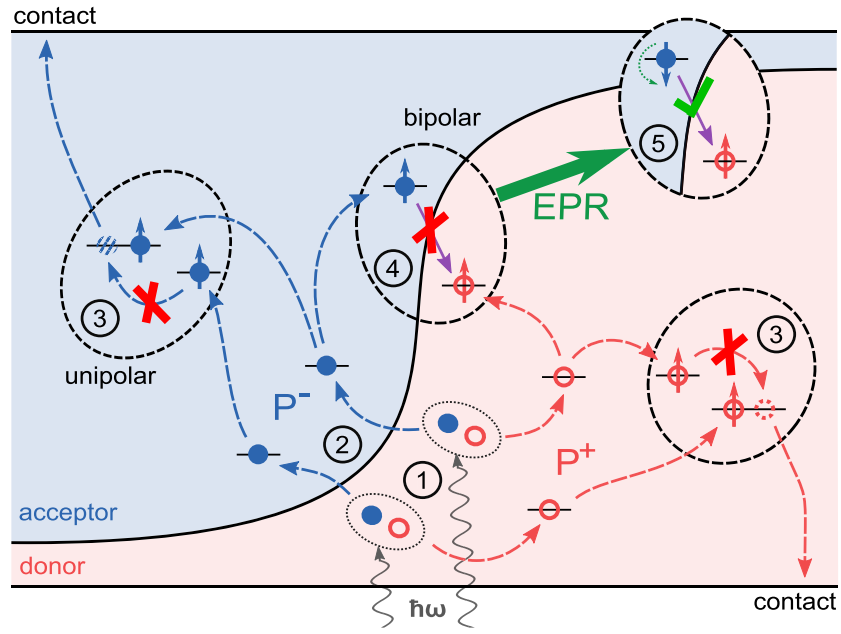

FIG. 1. (Color online) Schematic diagram of charge transport in bulk heterojunction devices. After absorption of photons [denoted by (1)], the excitons generated diffuse to the interface, where the charges are separated, eventually forming polarons $\mathrm{P}^{+}$and $\mathrm{P}^{-}$[denoted by (2)]. Spin-dependent processes involving these polarons can be either unipolar, where polarons of the same charge form a bipolaron [denoted by (3)], or bipolar, where two polarons of different charge annihilate [denoted by (4)]. The Pauli principle demands that both hopping and recombination are possible only if the spins of the two polarons are antiparallel; polarons with parallel spin orientation will not be allowed to undergo the hopping or recombination process. As sketched exemplarily for the recombination, the respective process can be enhanced by magnetic resonance, when the spin of either polaron is flipped and the parallel spin configuration is changed to an antiparallel one [denoted by (5)].

for less than $5 \mathrm{~min}$. In the spectrometer, the helium atmosphere of the cryostat prevents degradation.

The printed P3HT/PCBM samples were produced on a flexible 130- $\mu \mathrm{m}$-thick polyester substrate (Melinex ST506 from Dupont-Teijin). The solar cell stack was prepared in an inverted architecture using full roll-to-roll processing of all layers at high speed following a method similar to literature reports $[29,30]$. The device stack comprises six printed layers starting with a flexographically printed slanted silver comb front electrode structure [30], rotary-screenprinted semitransparent PEDOT:PSS, slot-die-coated zinc oxide, slot-die-coated P3HT: PCBM $(1: 1)$, rotary-screenprinted PEDOT:PSS, and, finally, a rotary-screen-printed oppositely slanted silver comb back electrode structure. The solar cells, which are produced on a much larger area than needed, were cut into smaller pieces to fit into the spectrometer.

The samples for the LEPR measurement were blends of P3HT/PCBM (1:1) drop cast on glass; thus they were no working solar cells. These samples were sealed in a glass tube under inert gas.

\section{B. Experimental setup}

The pEDMR measurements were performed at $10 \mathrm{~K}$ and under a negative bias voltage of $-3 \mathrm{~V}$ in a custombuilt pEDMR spectrometer based on a commercial pulsed $X$-band resonator. At this temperature, all solar cells tested behaved as a photoconductor. Microwaves from two separate sources were shaped to rectangular pulses using mixers and a multichannel pulse generator and then amplified by a traveling-wave-tube amplifier. Photocurrent transients were amplified by transimpedance and low-noise voltage amplifiers, bandpass filtered, and recorded with a digitizer card. Boxcar integration of the transients (typically from 1.5 to $15 \mu \mathrm{s}$ ) generated a charge $\Delta Q$, the primary pEDMR signal [22,23]. Four-cycle phase cycling was used in all experiments based on echo sequences, cycling the phase of the leading and trailing $\pi / 2$ pulses by $180^{\circ}$ [31]. LEPR measurements were performed at $50 \mathrm{~K}$ in a commercial EPR spectrometer. The cwEDMR measurements were performed at $10 \mathrm{~K}$ in the same spectrometer. The samples were illuminated by continuous red LED light throughout most magnetic resonance experiments.

\section{EXPERIMENTS AND DISCUSSION}

\section{A. Spin-coated P3HT/PCBM heterojunctions}

\section{Initial experiments}

The negative and positive polarons $\mathrm{P}^{-}$and $\mathrm{P}^{+}$in $\mathrm{PCBM}$ and P3HT exhibit $g$ factors of 1.9996 and 2.0017, respectively, as observed by LEPR [32,33], transient EPR (trEPR) [34,35], and transient EDMR (trEDMR) [18]. For typical linewidths of $0.9 \mathrm{mT}$, these $g$ factors allow a near-perfect spectroscopic separation at $X$-band frequencies around $9 \mathrm{GHz}$, as desired for our study.

Figure 2 shows a comparison of the signatures of the two polarons as observed by us as a function of the static magnetic field $B_{0}$ with (i) LEPR at $50 \mathrm{~K}$, where the magnetization of the sample is measured, (ii) cwEDMR at $10 \mathrm{~K}$, where the $\mathrm{dc}$ photocurrent through the sample is detected, and (iii) pulsed EDMR also at $10 \mathrm{~K}$, where the photocurrent transient after a microwave pulse is investigated. In all cases illumination was performed with the red light of a LED. LEPR and cwEDMR are performed with the help of magnetic field

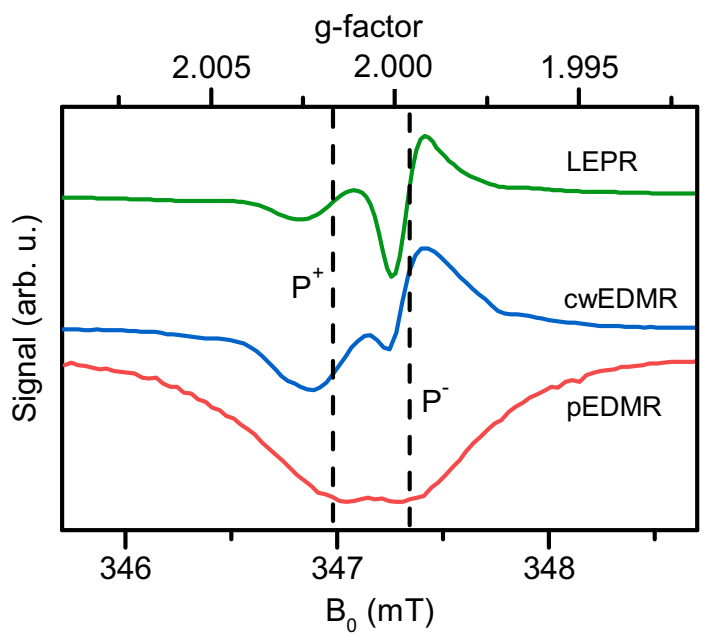

FIG. 2. (Color online) Light-induced electron paramagnetic resonance (LEPR), cw electrically detected magnetic resonance (cwEDMR), and pulsed EDMR (pEDMR) spectra of P3HT/PCBM. All spectra exhibit the characteristic resonances at the $g$ values of the positive and the negative polarons in P3HT and PCBM, respectively (depicted by vertical dashed lines). 
(a)

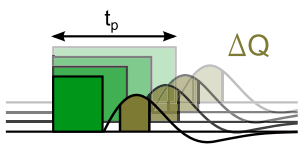

(b)

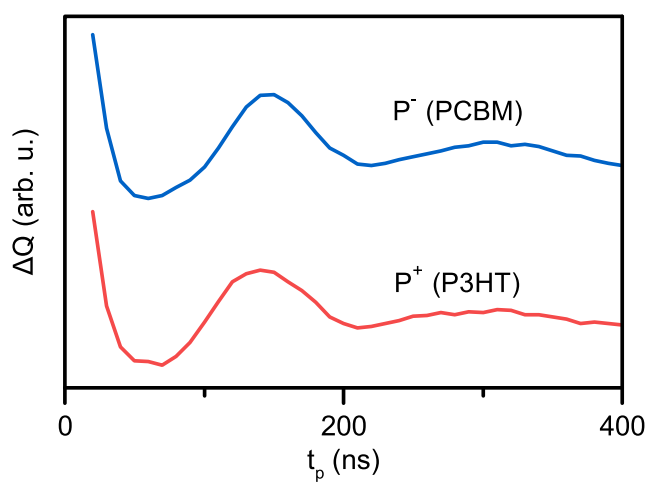

FIG. 3. (Color online) (a) Pulse sequence for the electrical detection of the coherent driving of spin nutations (Rabi oscillations). The current transients following the resonant manipulation of the spin system are integrated, yielding a charge $\Delta Q$ as the EDMR signal. (b) Electrically detected Rabi oscillations in P3HT/PCBM as a function of the microwave pulse length $t_{p}$ used to drive the magnetic resonance of the negative or positive polaron.

modulation, generating first-derivative spectra. As expected, in all these experiments the two different polarons are clearly and distinctly observed. In particular, all three types of EDMR experiments (cw, transient [18], and pulsed) on P3HT/PCBM heterojunctions show the participation of both polarons in the electric transport through the device. Still, neither the experiments summarized in Fig. 2 nor those of Kraffert et al. [18] can distinguish between bipolar polaron pair formation and unipolar bipolaron formation.

\section{Spin locking}

We will now use two more advanced pEDMR methods to identify the relevant pair process in bulk heterostructures based on single- and multifrequency spectroscopy.

Figure 3(b) shows the results of a Rabi oscillation experiment in pEDMR with the pulse sequence sketched in Fig. 3(a), where the length $t_{p}$ of the microwave pulse is varied. For both the positive and the negative polarons, virtually identical oscillations with a period of $\sim 130$ ns are observed.

By repeating these experiments with different microwave powers or at different $B_{0}$, we can distinguish between polaron pair formation and bipolaron formation using single-frequency spectroscopy based on the so-called spin locking. Spin locking occurs if the spectral excitation width is large enough to flip both spins belonging to a spin pair at the same time $[15,36]$. This is the case when the spectral width (i) becomes comparable to the inhomogeneous linewidth of the resonance in the case of bipolaron formation or (ii) becomes so large that both resonances can be excited in the opposite case of polaron pair formation. Since in EDMR the singlet symmetry of the polaronic spin pair is measured [37], the simultaneous driving of magnetic resonance on both constituents of the spin pair leads to an effective doubling of the Rabi oscillation, so that its frequency $\Omega=2 \gamma B_{1}$, where $\gamma$ is the gyromagnetic ratio and $B_{1}$ is the microwave field. For weak $B_{1}$, however, only one of the spins will be driven, so that in this case we expect the classic $\Omega=\gamma B_{1}$. This limit also enables the determination of $B_{1}$, which scales to higher microwave powers $P$ as $B_{1} \propto \sqrt{P}$.

The distinction between polaron pair formation and bipolaron formation can now be made easily if the two polaron resonances are spectrally separated: In the case of hopping between polarons of identical charge, the spin-locking signal at high $B_{1}$ will appear at the same spectral position as that of the polarons, while the corresponding signal for recombination involving polarons of different charge will be observed at magnetic fields between the resonance positions of the positively and negatively charged polarons. This is indeed observed in P3HT/PCBM. Figures 4(a) and 4(b) show Fourier transforms of Rabi oscillation experiments for different magnetic fields at two different microwave powers (below, we will refer to this type of plot as a Rabi map). Already for low $B_{1}$ [Fig. 4(a)], a small trace of spin locking is visible between the resonances of the polarons in P3HT and PCBM, as expected for bipolar polaron pairs. For higher microwave intensities [Fig. 4(b)] this spin-locking peak becomes even more prominent.

This assignment is further supported by simulation. Following the approach of Limes et al., the spin Hamiltonian is solved numerically using the superoperator Liouville space formalism [38]. Assuming an exchange interaction $J / 2 \pi$ of $2 \mathrm{MHz}$ between the positive and negative polarons, Larmor frequencies corresponding to the $g$ factors given above, and a $B_{1}$ of $0.38 \mathrm{mT}$, the Rabi frequency map shown in Fig. 4(c) is obtained. In addition, a Gaussian distribution of Larmor frequency differences of $25 \mathrm{MHz}$ is used to account for the inhomogeneous linewidths of $\sim 0.9 \mathrm{mT}$ of both polarons at $X$-band frequencies. The simulation reproduces the experimental Rabi maps well, particularly with respect to the central bipolar spin-locking feature at $\Omega / 2 \pi \approx 22 \mathrm{MHz}$. The parabolalike wings visible in both experiment and simulation are caused by the fact that Rabi oscillations speed up off resonance [23]. The exact line shapes of the polarons, variations in their coupling, and details of the microwave pulse shape and the effects of the resonator are not taken into account in the simulation, likely causing the remaining differences between simulation and experiment.

The additional feature in the fast Fourier transform spectra in Fig. 4(b) at $\Omega / 2 \pi \approx 14.8 \mathrm{MHz}$ can be attributed to hyperfine interaction with hydrogen nuclear spins. This interaction gives rise to additional oscillations which persist even after the Rabi oscillations have dephased. These oscillations do not change their period with the microwave $B_{1}$ field, but a clear dependence of the frequency on the static magnetic field $B_{0}$ is visible [Fig. 4(d)]. This dependence allows attribution to a nuclear magnetic resonance with a $g$ factor of 5.56(4), which is in very good agreement with the proton $g$ factor [39] and previous findings on PPV [40].

\section{Double resonance}

Electron double resonance (ELDOR) [24] and double electron-electron resonance (DEER) [25] experiments using pulse sequences, where the recombination partners are addressed separately by different microwave frequencies, show even more convincingly that bipolar polaron pair recombina- 
(a)

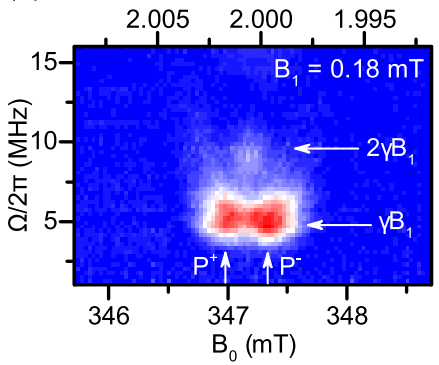

(b)

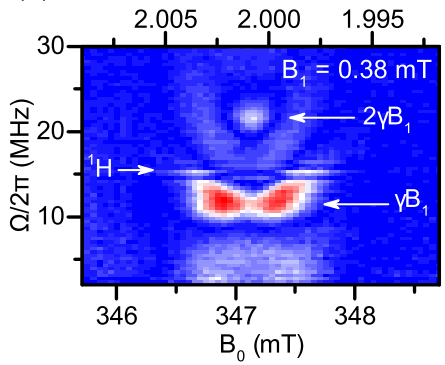

(c)

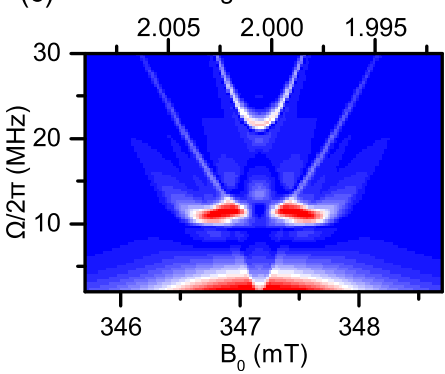

(d)

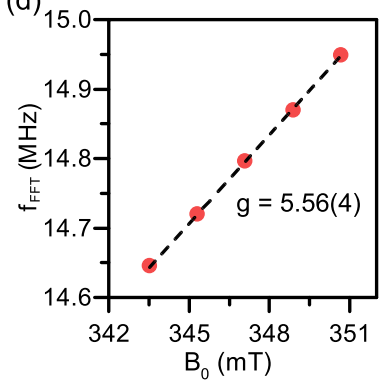

FIG. 4. (Color online) (a) Fast Fourier transforms of Rabi oscillation measured in P3HT/PCBM by pEDMR for different magnetic fields $B_{0}$ at a low microwave power. Two strong signals attributed to $\mathrm{P}^{+}$and $\mathrm{P}^{-}$are observed at the fundamental Rabi frequency $\Omega=\gamma B_{1}$. Already a weak spin-locking signal with $\Omega=2 \gamma B_{1}$ is visible at a central magnetic field. (b) At higher microwave power, the spin-locking signal becomes more prominent. In addition, a nuclear magnetic resonance signal with a frequency of $\sim 14.8 \mathrm{MHz}$ emerges, caused by hyperfine interaction with ${ }^{1} \mathrm{H}$ protons in the organic material [see (d)]. (c) Simulation of the Rabi map in (b), using a superoperator Liouville space formalism and assuming an exchange coupling between the positive and negative polarons of $J / 2 \pi=2 \mathrm{MHz}$. (d) The frequency of the horizontal features in (b) depends linearly on the magnetic field $B_{0}$, with a proportionality characteristic for the nuclear magnetic resonance of protons.

tion is observed. The results for the spin-coated P3HT/PCBM cells obtained with ELDOR are summarized in Figs. 5(a) and 5 (b). The experiments were performed at a fixed $B_{0}$, with one of the microwave frequencies tuned to resonantly excite the positive polaron and the other tuned to excite the negative counterpart. First, a Rabi oscillation experiment is performed as in Fig. 3, here on the $\mathrm{P}^{+}$, plotted in Fig. 5(b). The pEDMR signal initially decreases from a level indicative of the spins in the polaron pair being parallel to each other to a level corresponding to antiparallel spins due to a $\pi$ inversion pulse on one of the spins, $\mathrm{P}^{+}$. If, preceding this Rabi experiment, a $\pi$ pulse is applied to the other spin, $\mathrm{P}^{-}$in this case, the Rabi oscillation will start in an antiparallel pair configuration, turning into a parallel configuration after a pulse of length $\pi$

(a)

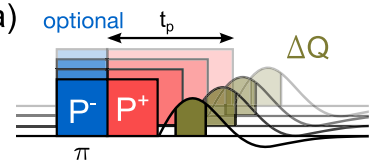

(b)

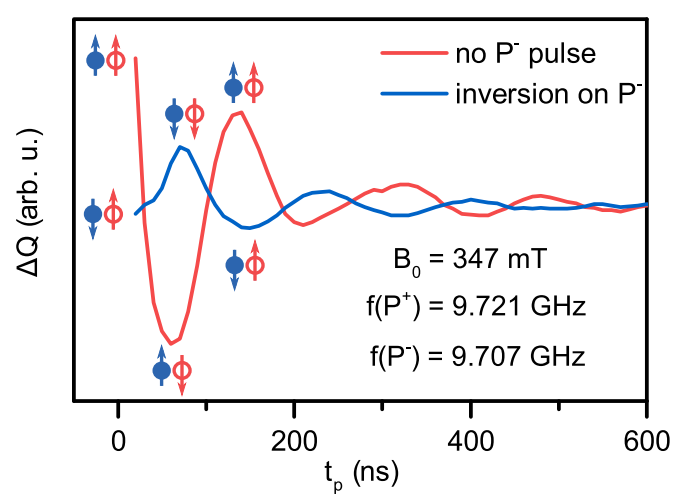

FIG. 5. (Color online) (a) Pulse sequence for electron double resonance (ELDOR) using two microwave frequencies to address the positive and negative polarons for a certain magnetic field $B_{0}$ separately. (b) Without a leading pulse on $\mathrm{P}^{-}$, a Rabi oscillation experiment on $\mathrm{P}^{+}$leads to the same oscillation already observed in Fig. 3(b). With a leading $\pi$ pulse inverting the $\mathrm{P}^{-}$spin, the oscillation in $\mathrm{P} 3 \mathrm{HT} / \mathrm{PCBM}$ is inverted, as expected for bipolar polaron pair recombination. for the $\mathrm{P}^{+}$Rabi experiment. The result will be an inversion of the Rabi oscillation, which is indeed observed in Fig. 5(b). If, on the other hand, unipolar hopping were to be observed, the spin symmetry of $\mathrm{P}^{+} \mathrm{P}^{+}$pairs observed in the Rabi oscillation would remain the same irrespective of changes of the spin state of $\mathrm{P}^{-}$, which is not observed. The degree of inversion in the bipolar case depends on the degree to which the $\mathrm{P}^{-}$ensemble is addressed by the corresponding microwave pulse, which is about $70 \%$ in our case.

These ELDOR experiments can also be performed as a function of the microwave frequencies used. The pulse sequence of such an ELDOR Rabi map is shown in Fig. 6(a). With a frequency $f_{\text {pump }}$ and for varying times $t_{\text {pump }}$ a

(a)

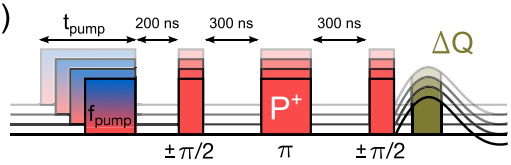

(b)

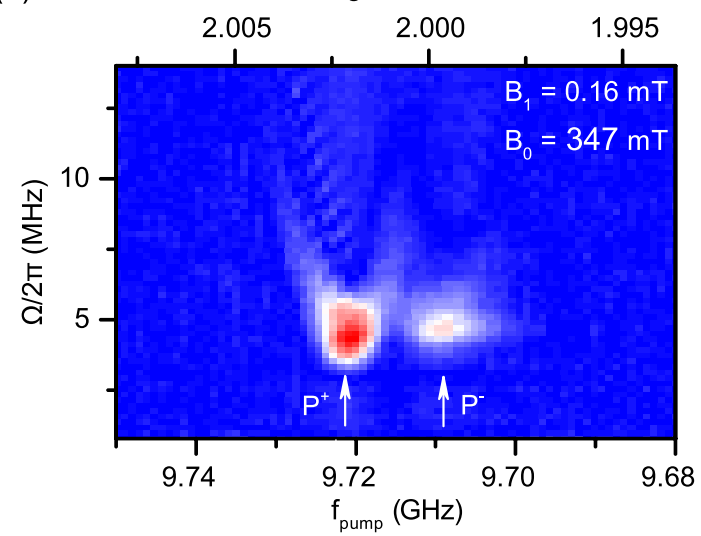

FIG. 6. (Color online) (a) Modification of the ELDOR pulse sequence for frequency mapping. A pulse of length $t_{\text {pump }}$ and frequency $f_{\text {pump }}$ is followed by an echo sequence on the $\mathrm{P}^{+}$resonance to measure the influence of the first pulse on the positive polaron. (b) Corresponding ELDOR Rabi map for P3HT/PCBM. A clear and spectrally well resolved Rabi oscillation driving the $\mathrm{P}^{-}$resonance is observed on $\mathrm{P}^{+}$. 
(a)

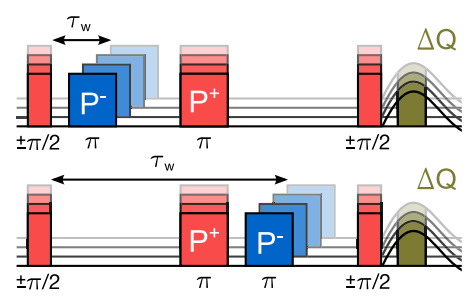

(b)

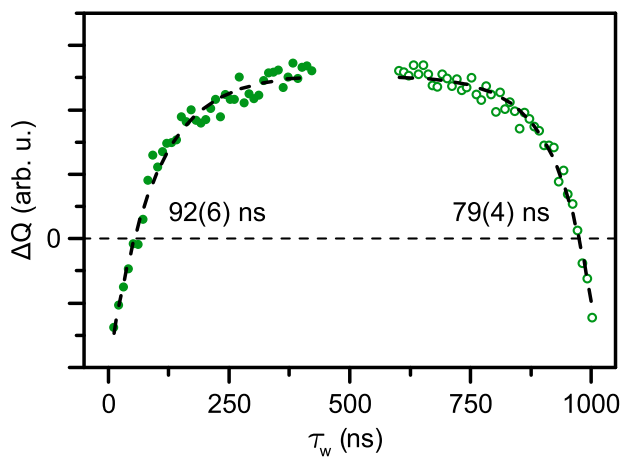

FIG. 7. (Color online) (a) Pulse sequence for the determination of the spin coupling strength via double electron-electron resonance (DEER). (b) Echo intensity as a function of the delay between the initial $\pi / 2$ pulse on $\mathrm{P}^{+}$and the $\pi$ inversion pulse on $\mathrm{P}^{-}$ in $\mathrm{P} 3 \mathrm{HT} / \mathrm{PCBM}$. The time dependence allows us to estimate the coupling strength to $\sim 2 \mathrm{MHz}$.

microwave pulse is applied to the P3HT/PCBM cell, followed by an echo sequence on the $\mathrm{P}^{+}$resonance used to measure the $\mathrm{P}^{+}$spin state [41]. With respect to the pulse sequence in Fig. 5(a), this is effectively only a time-inverted experiment but allows us to use phase cycling and lock-in detection [31], very significantly improving the signal-to-noise ratio. The corresponding ELDOR Rabi map is shown in Fig. 6(b). As in the case of the single-frequency Rabi map, a signal is seen in the Fourier transform corresponding to $\Omega=\gamma B_{1}$. The signal is strong when $f_{\text {pump }}$ also excites $\mathrm{P}^{+}$and, in agreement with Fig. 5(b), somewhat weaker when exciting $\mathrm{P}^{-}$. Most importantly, there is a clear signal minimum between the two resonances, even more than in Fig. 4(a), which shows the corresponding single-frequency Rabi map. Spurious excitation of $\mathrm{P}^{+}$in the experiments of Figs. 5(a) and 5(b) due to the small spectral overlap of $\mathrm{P}^{+}$and $\mathrm{P}^{-}$can therefore be excluded.

While the ELDOR experiments discussed show that, indeed, bipolar spin pairs are formed, DEER experiments are able to quantitatively estimate the strength of the coupling between the two polarons. The corresponding pulse sequence is shown in Fig. 7(a), where an inversion pulse on $\mathrm{P}^{-}$is applied either in the first or the second evolution period of an echo sequence on $\mathrm{P}^{+}$. The change in the magnetic environment of $\mathrm{P}^{+}$during its evolution period due to the change of the coupled spin state of $\mathrm{P}^{-}$will lead to an inversion of the signal depending on the point in time when the $\mathrm{P}^{-}$spin is flipped. While, ideally, a well-defined spin-spin interaction manifests itself in an oscillatory behavior of the echo signal as a function of the shift $\tau_{w}$ [see Fig. 7(a)], a broad distribution of coupling strengths typically leads to an exponential decay of the echo intensity, still allowing us to estimate a characteristic coupling from the decay constant. The results of the DEER experiments on the P3HT/PCBM cells are shown in Fig. 7(b), with characteristic decay times of 92 and 79 ns in the case of the inversion pulse in the first and second evolution times, respectively, corresponding to a typical coupling strength of $2 \mathrm{MHz}$ between the negative and positive polarons. This value was already used in the simulation in Fig. 4(c). The lack of oscillation in the DEER results indicates a broad distribution of coupling strengths, which was not taken into account in the simulation and is one of the reasons for the remaining differences between experiment and simulation in Fig. 4.

Nevertheless, both types of dual-frequency experiments, ELDOR and DEER, performed here clearly support the initial argument, based on the single-frequency spin-locking measurements, that spin-dependent processes in bipolar polaron pairs are observed. Since cwEDMR experiments without lock-in amplification (data not shown) show a reduction of the photocurrent under the resonance condition, we can attribute the resonances observed to bipolar polaron pair recombination since unipolar bipolaron hopping would lead to a resonant increase of the mobility and therefore the conductivity.

\section{Lifetimes and coherence times}

The observation that the dominant spin-dependent process in the P3HT/PCBM solar cells at low temperatures is the bipolar polaron pair recombination allows us to transfer a wealth of pulse sequences for the study of spin and recombination kinetics, which were mostly developed to investigate the recombination involving donors and defects in crystalline Si [41,42], also to organic semiconductors. In particular, inversion recovery experiments can be used to determine the recombination times of polaron pairs with antiparallel and parallel spin orientations $\tau_{\mathrm{ap}}$ and $\tau_{\mathrm{p}}$, respectively [Fig. 8(a)]. The steady-state spin population during illumination, which, due to the fast spin-allowed recombination of antiparallel spin pairs, mostly consists of parallel spin pairs, is first inverted into antiparallel pairs by a $\pi$ inversion pulse. The recombination of those pairs is then measured with an echo sequence. To prohibit photogeneration of new charge carriers before the readout, inversion recovery experiments are the only pEDMR measurements reported here where the LED illumination is switched off $(50 \mu \mathrm{s}$ before the start of the microwave sequence). As expected for pair recombination, the corresponding experiments performed on the positive and negative polarons agree, with $\tau_{\mathrm{ap}} \approx 74 \mu \mathrm{s}$ [Fig. 8(b)]. Since the excitation width is again not large enough to invert the whole ensemble, parallel spin pairs persist; their recombination time $\tau_{\mathrm{p}} \approx 3 \mathrm{~ms}$ can therefore be determined in the same experiment. Due to the variation in the coupling strengths, the recombination kinetics are best described by stretched exponentials $\exp \left[-(t / \tau)^{n}\right]$ (see Ref. [41]). In our experiment, we observe $n_{\text {ap }}=0.45$ and $n_{\mathrm{p}}=0.25$ for the recombination of antiparallel and parallel spins, respectively.

The echo sequence can also be used to measure the decoherence time of the polaron spins [Fig. 8(c)]. We find values of $T_{2}=1.9 \mu \mathrm{s}$ for the positive polaron in P3HT and $T_{2}=1.5 \mu \mathrm{s}$ for the negative counterpart in PCBM [Fig. 8(d)], obtained by fitting with normal exponential functions. 
(a)

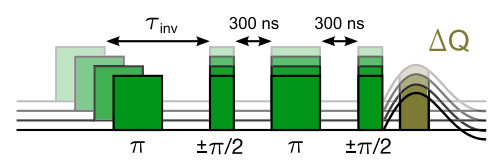

(b)

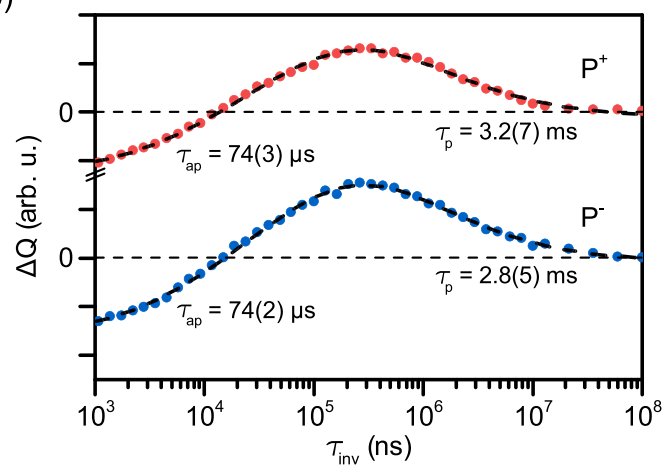

(c)

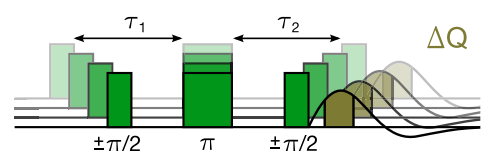

(d)

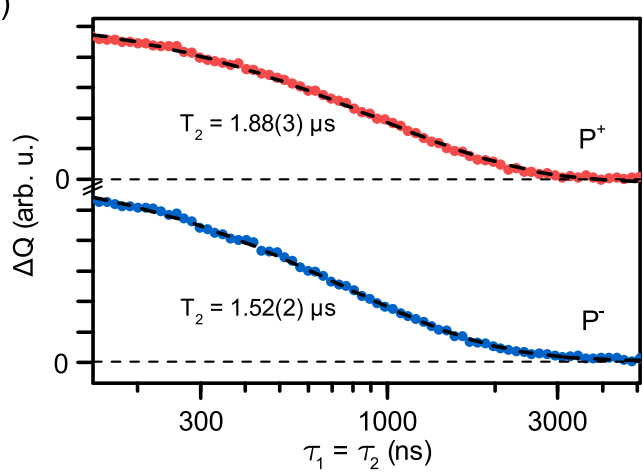

FIG. 8. (Color online) (a) Pulse sequence used to determine the antiparallel and the parallel recombination times $\tau_{\text {ap }}$ and $\tau_{\mathrm{p}}$, respectively, of $\mathrm{P}^{+} \mathrm{P}^{-}$polaron pairs based on an inversion recovery experiment. (b) Typical time constants of $74 \mu \mathrm{s}$ for $\tau_{\text {ap }}$ and $3 \mathrm{~ms}$ for $\tau_{\mathrm{p}}$ are found experimentally in P3HT/PCBM at $10 \mathrm{~K}$. (c) Standard echo sequence including a final $\pi / 2$ projection pulse for the measurement of the coherence time $T_{2}$. (d) Both polarons exhibit similar decoherence properties in P3HT/PCBM with $T_{2} \approx 1.7 \mu \mathrm{s}$.

\section{B. Printed P3HT/PCBM heterojunctions}

In order to compare the pEDMR results for differently processed samples, the Rabi measurements were repeated on roll-to-roll-produced P3HT/PCBM solar cells [43]. These cells show essentially the same spectral and Rabi oscillation behaviors, including a clear spin-locking signature (Fig. 9). Therefore, the specific production process seems to have a negligible influence on the results of our experiments, allowing us to conclude that bipolar recombination is the dominant spin-dependent process in $\mathrm{P} 3 \mathrm{HT} / \mathrm{PCBM}$ structures at low temperatures. The only significant difference between spincoated and roll-to-roll printed devices is the lower pEDMR signal intensity of the latter, notable in the lower signal-tonoise ratio realizable in the experiments, an indication that less recombination takes place in these optimized devices.

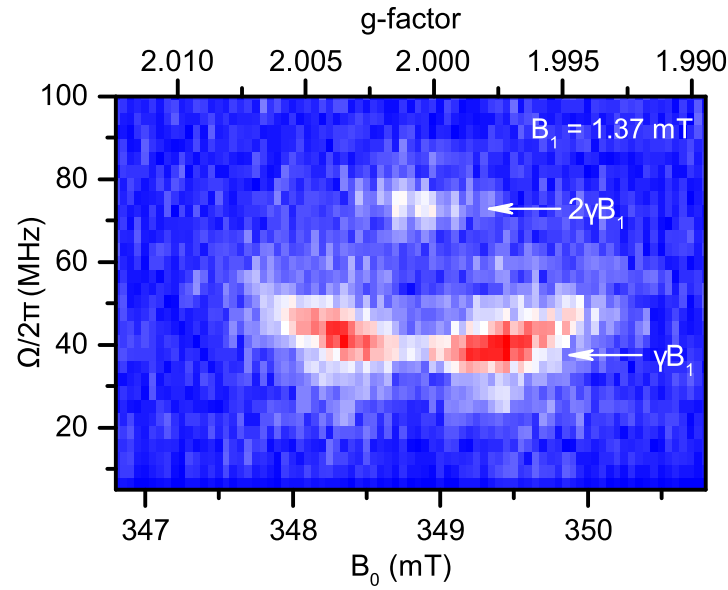

FIG. 9. (Color online) Fast Fourier transform of Rabi oscillation measurements by $\mathrm{pEDMR}$ on printed $\mathrm{P} 3 \mathrm{HT} / \mathrm{PCBM}$ heterojunctions at $10 \mathrm{~K}$. Again, the two signals corresponding to $\mathrm{P}^{+}$and $\mathrm{P}^{-}$are observed, with the central spin-locking signal appearing at high microwave powers.

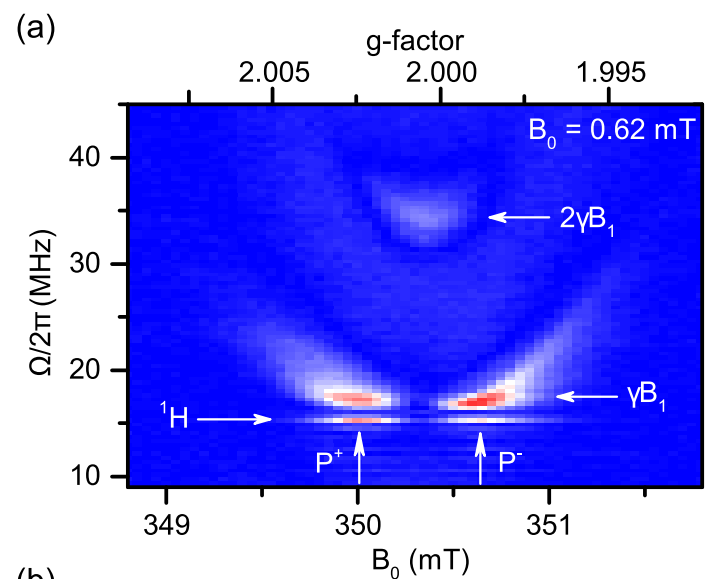

(b)

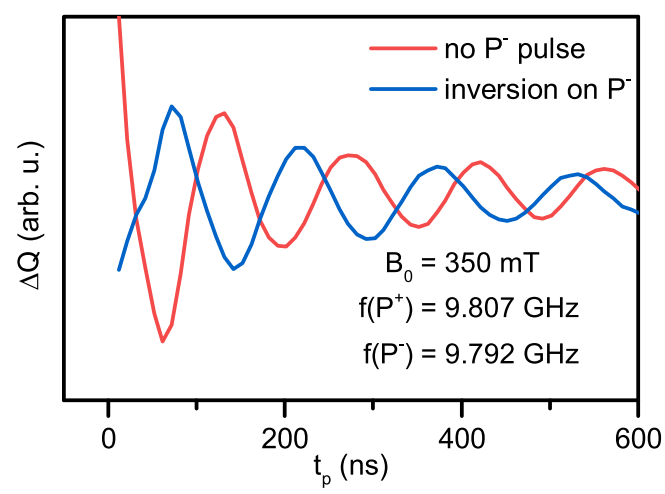

FIG. 10. (Color online) (a) Fast Fourier transform of Rabi oscillations in a spin-coated PCDTBT/PCBM solar cell at $10 \mathrm{~K}$. Analogous to the $\mathrm{P} 3 \mathrm{HT} / \mathrm{PCBM}$ cells, signals corresponding to $\mathrm{P}^{+}$and $\mathrm{P}^{-}$and a central spin-locking signal are observed. The feature at $\sim 15 \mathrm{MHz}$ is again attributed to hyperfine interaction with hydrogen nuclei. (b) Rabi oscillations on $\mathrm{P}^{+}$and the ELDOR experiment with a leading $\pi$ pulse on $\mathrm{P}^{-}$in the PCDTBT/PCBM solar cell. As with $\mathrm{P} 3 \mathrm{HT} / \mathrm{PCBM}$, the Rabi oscillations are inverted in the ELDOR experiment on PCDTBT/PCBM, as expected for bipolar polaron pair recombination. 


\section{Spin-coated PCDTBT/PCBM heterojunctions}

To test the generality of the results obtained so far on at least a second polymer/fullerene heterojunction, we have repeated the above experiments on PCDTBT/PCBM solar cells. This combination is suited for such studies since the positive polaron $\mathrm{P}^{+}$on PCDTBT with $g=2.0025$ (average of anisotropic $g$ values) [44] can similarly be spectrally resolved from $\mathrm{P}^{-}$on PCBM as in the case of P3HT/PCBM. In full agreement with the results of the P3HT/PCBM solar cells, the Rabi map shows the typical features of bipolar polaron pair recombination [Fig. 10(a)]: the two peaks at a frequency of $\gamma B_{1}$ belonging to the polarons $\mathrm{P}^{+}$and $\mathrm{P}^{-}$and a third central peak due to spin locking. In the corresponding ELDOR experiment the Rabi oscillation is again clearly inverted [Fig. 10(b)]. Both the spin-locking signals in the Rabi map and the ELDOR inversion demonstrate that in PCDTBT/PCBM heterojunctions bipolar polaron recombination is also the dominant spin-dependent process at low temperature and under illumination. Echo decay and inversion recovery experiments reveal a spin coherence time of $T_{2}=4.2 \mu \mathrm{s}$ and antiparallel and parallel recombination times of $\tau_{a p}=93 \mu \mathrm{s}$ and $\tau_{p}=3.9 \mathrm{~ms}$, respectively (data not shown), which are very similar to the $\mathrm{P} 3 \mathrm{HT} / \mathrm{PCBM}$ results.

\section{CONCLUSIONS}

We have shown that pulsed EDMR can be used to distinguish between bipolar polaron pair recombination and unipolar bipolaron hopping transport in organic semiconductors when the resonant signatures of the differently charged polarons can be separated spectroscopically. The fact that bipolar polaron pair recombination is observed in two different polymer/fullerene heterostructures suggests that this spindependent process is rather general to organic diodes, at least at low temperatures. The assignment to bipolar recombination is made on the basis of different single- and multifrequency experiments, including spin locking, ELDOR, and DEER. In addition to the identification of the dominant spin-dependent process, the advanced pulse sequences used allow the determination of the strength of the coupling between the polarons, the lifetimes of polaron pairs with antiparallel and parallel spin orientations, and the coherence time of the spins. The pEDMR experiments performed here are highly versatile and were applied to both laboratory-scale devices and cells fabricated on an industrial scale, demonstrating the generality of the spin-dependent process identified. This method is limited to neither the material systems nor the $10 \mathrm{~K}$ temperature range investigated here. In particular, optical excitation with nanosecond pulses might allow us to study the temporal evolution of the polaron coupling, effectively monitoring the formation of the polaron pairs, or even to investigate chargetransfer levels with pEDMR methods. Optical excitation resonant with the charge-transfer levels will be helpful in this respect [45]. Therefore, the present work could be an important stepping stone for further investigations of related material systems to understand charge-transport processes as well as the microscopic steps involved in photovoltaic energy conversion and lighting applications in organic devices.

\section{ACKNOWLEDGMENTS}

This work was supported by the Eurotech Alliance through the International Graduate School of Science and Engineering (IGSSE), project Interface Science for Photovoltaics (ISPV). The authors thank W. Aigner, D. Franke, A. Greppmair, F. Hrubesch, A. Sperlich, M. Suckert, and S. Väth for helpful discussions.
[1] S. Reineke, F. Lindner, G. Schwartz, N. Seidler, K. Walzer, B. Lüssem, and K. Leo, Nature (London) 459, 234 (2009).

[2] Z. Xu and B. Hu, Adv. Funct. Mater. 18, 2611 (2008).

[3] D. M. González, V. Kärstgens, Y. Yao, L. Song, G. Santoro, S. V. Roth, and P. Müller-Buschbaum, Adv. Energy Mater. 5, 1401770 (2015).

[4] J.-W. Yoo, H. W. Jang, V. N. Prigodin, C. Kao, C. B. Eom, and A. J. Epstein, Synth. Met. 160, 216 (2010).

[5] C. Boehme and J. M. Lupton, Nat. Nanotechnol. 8, 612 (2013).

[6] E. L. Frankevich, M. M. Tribel, I. A. Sokolik, and A. I. Pristupa, Phys. Status Solidi 87, 373 (1978).

[7] L. S. Swanson, J. Shinar, A. R. Brown, D. D. C. Bradley, R. H. Friend, P. L. Burn, A. Kraft, and A. B. Holmes, Phys. Rev. B 46, 15072 (1992).

[8] V. Dyakonov, N. Gauss, G. Rösler, S. Karg, W. Rieß, and M. Schwoerer, Chem. Phys. 189, 687 (1994).

[9] N. C. Greenham, J. Shinar, J. Partee, P. A. Lane, O. Amir, F. Lu, and R. H. Friend, Phys. Rev. B 53, 13528 (1996).

[10] M. S. Brandt and M. Stutzmann, Bull. Am. Phys. Soc. 38, 560 (1993).

[11] T. Eickelkamp, S. Roth, and M. Mehring, Mol. Phys. 95, 967 (1998).
[12] E. L. Frankevich, A. A. Lymarev, I. Sokolik, F. E. Karasz, S. Blumstengel, R. H. Baughman, and H. H. Hörhold, Phys. Rev. B 46, 9320 (1992).

[13] K. Schulten, H. Staerk, A. Weller, H.-J. Werner, and B. Nickel, Z. Phys. Chem. 101, 371 (1976).

[14] P. A. Bobbert, T. D. Nguyen, F. W. A. van Oost, B. Koopmans, and M. Wohlgenannt, Phys. Rev. Lett. 99, 216801 (2007).

[15] J. Behrends, A. Schnegg, K. Lips, E. A. Thomsen, A. K. Pandey, I. D. W. Samuel, and D. J. Keeble, Phys. Rev. Lett. 105, 176601 (2010).

[16] K. J. van Schooten, D. L. Baird, M. E. Limes, J. M. Lupton, and C. Boehme, Nat. Commun. 6, 6688 (2015).

[17] J. Behrends, I. D. W. Samuel, A. Schnegg, and D. J. Keeble, Nat. Nanotechnol. 8, 884 (2013).

[18] F. Kraffert, R. Steyrleuthner, C. Meier, R. Bittl, and J. Behrends, Appl. Phys. Lett. 107, 043302 (2015).

[19] C. Boehme, D. R. McCamey, K. J. van Schooten, W. J. Baker, S.-Y. Lee, S.-Y. Paik, and J. M. Lupton, Phys. Status Solidi 246, 2750 (2009).

[20] W. J. Baker, D. R. McCamey, K. J. van Schooten, J. M. Lupton, and C. Boehme, Phys. Rev. B 84, 165205 (2011). 
[21] B. Z. Tedlla, F. Zhu, M. Cox, J. Drijkoningen, J. Manca, B. Koopmans, and E. Goovaerts, Adv. Energy Mater. 5, 1401109 (2014).

[22] C. Boehme and K. Lips, Phys. Rev. B 68, 245105 (2003).

[23] A. R. Stegner, C. Boehme, H. Huebl, M. Stutzmann, K. Lips, and M. S. Brandt, Nat. Phys. 2, 835 (2006).

[24] F. Hoehne, H. Huebl, B. Galler, M. Stutzmann, and M. S. Brandt, Phys. Rev. Lett. 104, 046402 (2010).

[25] M. Suckert, F. Hoehne, L. Dreher, M. Kuenzl, H. Huebl, M. Stutzmann, and M. S. Brandt, Mol. Phys. 111, 2690 (2013).

[26] D. Chi, S. Qu, Z. Wang, and J. Wang, J. Mater. Chem. C 2, 4383 (2014).

[27] J. Liu, Q. Liang, H. Wang, M. Li, Y. Han, Z. Xie, and L. Wang, J. Phys. Chem. C 118, 4585 (2014).

[28] D. R. McCamey, H. A. Seipel, S.-Y. Paik, M. J. Walter, N. J. Borys, J. M. Lupton, and C. Boehme, Nat. Mater. 7, 723 (2008).

[29] F. C. Krebs, M. Hösel, M. Corazza, B. Roth, M. V. Madsen, S. A. Gevorgyan, R. R. Søndergaard, D. Karg, and M. Jørgensen, Energy Technol. 1, 378 (2013).

[30] F. C. Krebs, N. Espinosa, M. Hösel, R. R. Søndergaard, and M. Jørgensen, Adv. Mater. 26, 29 (2014).

[31] F. Hoehne, L. Dreher, J. Behrends, M. Fehr, H. Huebl, K. Lips, A. Schnegg, M. Suckert, M. Stutzmann, and M. S. Brandt, Rev. Sci. Instrum. 83, 043907 (2012).

[32] V. I. Krinichnyi, E. I. Yudanova, and N. N. Denisov, J. Chem. Phys. 131, 044515 (2009).

[33] R. Dietmueller, A. R. Stegner, R. Lechner, S. Niesar, R. N. Pereira, M. S. Brandt, A. Ebbers, M. Trocha, H. Wiggers, and M. Stutzmann, Appl. Phys. Lett. 94, 113301 (2009).
[34] J. Behrends, A. Sperlich, A. Schnegg, T. Biskup, C. Teutloff, K. Lips, V. Dyakonov, and R. Bittl, Phys. Rev. B 85, 125206 (2012).

[35] J. Niklas, S. Beaupré, M. Leclerc, T. Xu, L. Yu, A. Sperlich, V. Dyakonov, and O. G. Poluektov, J. Phys. Chem. B 119, 7407 (2015).

[36] D. R. McCamey, K. J. van Schooten, W. J. Baker, S.-Y. Lee, S.-Y. Paik, J. M. Lupton, and C. Boehme, Phys. Rev. Lett. 104, 017601 (2010).

[37] D. Kaplan, I. Solomon, and N. F. Mott, J. Phys. Lett. 39, 51 (1978).

[38] M. E. Limes, J. Wang, W. J. Baker, S.-Y. Lee, B. Saam, and C. Boehme, Phys. Rev. B 87, 165204 (2013).

[39] P. J. Mohr, B. N. Taylor, and D. B. Newell, Rev. Mod. Phys. 84, 1527 (2012).

[40] H. Malissa, M. Kavand, D. P. Waters, K. J. van Schooten, P. L. Burn, Z. V. Vardeny, B. Saam, J. M. Lupton, and C. Boehme, Science 345, 1487 (2014).

[41] F. Hoehne, L. Dreher, M. Suckert, D. P. Franke, M. Stutzmann, and M. S. Brandt, Phys. Rev. B 88, 155301 (2013).

[42] S.-Y. Paik, S.-Y. Lee, W. J. Baker, D. R. McCamey, and C. Boehme, Phys. Rev. B 81, 075214 (2010).

[43] R. R. Søndergaard, M. Hösel, and F. C. Krebs, J. Polymer Sci., Part B: Polym. Phys. 51, 16 (2013).

[44] J. Niklas, K. L. Mardis, B. P. Banks, G. M. Grooms, A. Sperlich, V. Dyakonov, S. Beaupré, M. Leclerc, T. Xu, L. Yu, and O. G. Poluektov, Phys. Chem. Chem. Phys. 15, 9562 (2013).

[45] R. A. Street, K. W. Song, J. E. Northrup, and S. Cowan, Phys. Rev. B 83, 165207 (2011). 